PROCEEDINGS OF THE

AMERICAN MATHEMATICAL SOCIETY

Volume 137, Number 9, September 2009, Pages 3105-3114

S 0002-9939(09)09862-1

Article electronically published on April 23, 2009

\title{
COMPACT GRAPHS OVER A SPHERE OF CONSTANT SECOND ORDER MEAN CURVATURE
}

\author{
A. BARROS AND P. SOUSA
}

(Communicated by Richard A. Wentworth)

\begin{abstract}
The aim of this work is to show that a compact smooth starshaped hypersurface $\Sigma^{n}$ in the Euclidean sphere $\mathbb{S}^{n+1}$ whose second function of curvature $S_{2}$ is a positive constant must be a geodesic sphere $\mathbb{S}^{n}(\rho)$. This generalizes a result obtained by Jellett in 1853 for surfaces $\Sigma^{2}$ with constant mean curvature in the Euclidean space $\mathbb{R}^{3}$ as well as a recent result of the authors for this type of hypersurface in the Euclidean sphere $\mathbb{S}^{n+1}$ with constant mean curvature. In order to prove our theorem we shall present a formula for the operator $L_{r}(g)=\operatorname{div}\left(P_{r} \nabla g\right)$ associated with a new support function $g$ defined over a hypersurface $M^{n}$ in a Riemannian space form $M_{c}^{n+1}$.
\end{abstract}

\section{INTRODUCTION}

This paper continues work of the authors concerning constant mean curvature [5], which was inspired by a paper due to Jellett [11] published in the middle of the nineteenth century in which he proved that a smooth star-shaped constant mean curvature surface $\Sigma^{2} \subset \mathbb{R}^{3}$ is a round sphere. On the other hand, if $\Sigma^{n} \subset \mathbb{R}^{n+1}$ is an oriented hypersurface and $k_{1}, \ldots, k_{n}$ represent the principal curvatures of $\Sigma^{n}$, we may consider similar problems related to the $r^{\text {th }}$ elementary symmetric functions $S_{r}$ given by $S_{r}=\sum k_{i_{1}} \ldots k_{i_{r}}, r=0,1, \ldots, n$. For instance, Suss [18] proved that a compact convex hypersurface $\Sigma^{n}$ in the Euclidean space $\mathbb{R}^{n+1}$ with some $S_{r}$ constant must be a round sphere. The convexity condition was improved upon by Hsiung [10, who showed that a hypersurface $\Sigma^{n} \subset \mathbb{R}^{n+1}$ whose classical support function has a well-defined sign with any symmetric function of the principal curvatures constant must also be a round sphere. Later, Ros proved in [14] and [15] that a round sphere is the unique compact embedded hypersurface in Euclidean space if any symmetric function $S_{r}$ is constant. Subsequently, Montiel and Ros 12 . extended this result to any compact embedded hypersurface in the hyperbolic space $\mathbb{H}^{n+1}$ as well as to one contained in an open hemisphere of the Euclidean sphere $\mathbb{S}^{n+1}$. On the other hand, it is well known that a product of spheres produces hypersurfaces in the Euclidean sphere $\mathbb{S}^{n+1}$ with $S_{r}$ constant for any $r=1, \ldots, n$. Therefore, for hypersurfaces contained in the Euclidean sphere $\mathbb{S}^{n+1}$ we have a lot of examples with $S_{r}$ constant which are not round spheres. However, returning

Received by the editors August 7, 2007, and, in revised form, December 27, 2008.

2000 Mathematics Subject Classification. Primary 53C42, 53C45; Secondary 53C65.

Key words and phrases. $L_{r}$ operator, radial graph, constant scalar curvature.

The first author was partially supported by CNPq-BR.

The second author was partially supported by CAPES-BR. 
to Jellett's idea, we may obtain a similar result in the Euclidean sphere $\mathbb{S}^{n+1}$ for compact smooth star-shaped hypersurfaces whose second function of curvature is positive while avoiding the condition that it be contained in an open hemisphere. More precisely, we shall prove the following theorem.

Theorem 1.1. Let $\Sigma^{n} \subset \mathbb{S}^{n+1}$ be a compact smooth star-shaped hypersurface with $S_{2}$ constant and positive. Then $\Sigma^{n}$ is totally umbilical.

\section{Conformal Fields}

Given a vector field $V \in \chi(\bar{M})$ in a Riemannian manifold $\bar{M}$ and a $r$-covariant tensor field $\omega$, the Lie derivative of $\omega$ with respect to $V$ is defined by

$$
\left(\mathcal{L}_{V} \omega\right)\left(X_{1}, \ldots, X_{r}\right)=V\left(\omega\left(X_{1}, \ldots, X_{r}\right)\right)-\sum_{i=1}^{r} \omega\left(X_{1}, \ldots,\left[V, X_{i}\right], \ldots, X_{r}\right) .
$$

For instance, if $\omega=\langle$,$\rangle stands for the Riemannian metric of \bar{M}$, then

$$
\left(\mathcal{L}_{V}\langle,\rangle\right)(X, Y)=\left\langle\nabla_{X} V, Y\right\rangle+\left\langle X, \nabla_{Y} V\right\rangle .
$$

We say that $V \in \chi(\bar{M})$ is a conformal vector field if there exists $\psi \in \mathcal{D}(\bar{M})$, which is called the conformal factor of $V$, such that

$$
\mathcal{L}_{V}\langle,\rangle=2 \psi\langle,\rangle \text {. }
$$

A useful conformal vector field in a space form $M_{c}^{n+1}$ is the position vector with origin at a fixed point $p_{0} \in M_{c}^{n+1}$. It was introduced for non-Euclidean space forms by Heintze 9 according to the following: Let $d: M_{c}^{n+1} \rightarrow \mathbb{R}$ be the distance function defined by $d()=.\operatorname{dist}\left(., p_{0}\right)$. The position vector field over $M_{c}^{n+1}$ is given by $V=s(d) \nabla d$ where $s(t)$ is the solution of the differential equation $y^{\prime \prime}+c y=0$ subject to the initial conditions $y(0)=0$ and $y^{\prime}(0)=1$.

On the other hand Greene and $\mathrm{Wu}[8]$ have shown an important property concerning the Hessian form of $d$ which states that

$$
s(d)\left\langle D_{X} \nabla d, Y\right\rangle=s^{\prime}(d)(\langle X, Y\rangle-\langle\nabla d, X\rangle\langle\nabla d, Y\rangle),
$$

for any vector fields $X, Y \in \chi\left(M_{c}^{n+1}\right)$. Here and elsewhere $D$ stands for the Riemannian connection of $M_{c}^{n+1}$. From the last relation we conclude that $V$ is a conformal vector field with conformal factor $\psi=s^{\prime}(d)$.

\section{3. $L_{r}$ OF A SUPPORT FUNCTION}

In this section we compute the operator $L_{r}$ of a new support function defined on a hypersurface $M^{n}$ in a space form $M_{c}^{n+1}$. For generalized Robertson-Walker space a particular case appears in Alias and Colares 11. Given a conformal vector field $V$ in $M_{c}^{n+1}$ and an isometric immersion $x: M^{n} \rightarrow M_{c}^{n+1}$, the support function is defined on $M$ by

$$
g(p)=\langle V, N\rangle(x(p))
$$

where $N$ stands for a unit normal field to $M^{n}$. Whenever necessary we shall identify $p \in M^{n}$ with its image $x(p) \in M_{c}^{n+1}$.

We now consider on $M$ the second fundamental form $A$, the $r^{\text {th }}$ symmetric function of curvature $S_{r}$, the $r^{\text {th }}$ Newton tensor $P_{r}$ and the operator $L_{r}$. These Newton tensors are defined inductively according to $P_{0}=I$ and, for $1 \leq r \leq n$, $P_{r}=S_{r} I-A P_{r-1}$, whereas the operator $L_{r}: D(M) \rightarrow D(M)$ is given by

$$
L_{r}(f)=\operatorname{tr}\left(P_{r} \circ \mathrm{Hess}_{f}\right) \text {. }
$$


It is important to point out that for hypersurfaces in a space form $M_{c}^{n+1}$ Rosenberg showed in [16] that each $L_{r}$ takes a divergence form. More exactly, we have

$$
L_{r}(f)=\operatorname{div}\left(P_{r} \nabla f\right) .
$$

Moreover, under the above conditions we list some important properties concerning $S_{r}$ and $P_{r}$ that may be found in [13] and [16].

- $\operatorname{tr}\left(P_{r}\right)=(n-r) S_{r}$;

- $\operatorname{tr}\left(A P_{r}\right)=(r+1) S_{r+1}$;

- $\operatorname{tr}\left(A^{2} P_{r}\right)=S_{1} S_{r+1}-(r+2) S_{r+2}$;

- $\operatorname{tr}\left(P_{r} \circ D_{X} A\right)=\left\langle X, \nabla S_{r+1}\right\rangle$;

- $P_{r}$ is a self-adjoint operator whose eigenvalues are $\frac{\partial S_{r+1}}{\partial \lambda_{i}}$, where the $\lambda_{i}$ 's are the eigenvalues of $A$.

We point out that for $r=0$ the operator $L_{r}$ is the Laplacian $\Delta$. In this case a slight modification of the next theorem was obtained by many authors (see e.g. [2], [4], 5], 7] and [17]), whereas formulae for $L_{r} X$ and $L_{r} N$ can be found in Reilly [13] and Rosenberg [16, where $X$ stands for the position vector while $N$ is a unit normal vector field to $M$.

Theorem 3.1. Let $x: M^{n} \rightarrow M_{c}^{n+1}$ be an oriented isometric immersion with unit normal vector field $N$. If $V$ is a conformal vector field on $M_{c}^{n+1}$ and $g=\langle V, N\rangle$ represents the support function on $M^{n}$, then

$$
\begin{aligned}
L_{r}(g) & =-\left(S_{1} S_{r+1}-(r+2) S_{r+2}\right) g-c(n-r) S_{r} g \\
& -(n-r) S_{r} N(\psi)-(r+1) S_{r+1} \psi-\left\langle V, \nabla S_{r+1}\right\rangle,
\end{aligned}
$$

where $\psi$, which we identify with $\psi \circ x$, is the conformal factor of $V$.

Proof. Given $p \in x\left(M^{n}\right)$, let $\left\{e_{1}(p), \ldots, e_{n}(p)\right\} \subset T_{p} M$ be an orthonormal basis which diagonalizes $A$ at $p$ and whose associated eigenvalues are $\lambda_{1}, \ldots, \lambda_{n}$, respectively. Denote by $\left\{e_{1}, \ldots, e_{n}\right\}$ the geodesic frame that extends the above basis to a neighborhood of $p$ in $x\left(M^{n}\right)$. We may do this on a neighborhood of $p$ in $M_{c}^{n+1}$ in such a way that $D_{N} e_{i}(p)=0$. We now denote by $\langle$,$\rangle the Riemannian metric of$ $M_{c}^{n+1}$ and use the short notation $\mathcal{L}_{N, N}=\left(\mathcal{L}_{V}\langle\rangle,\right)(N, N), \mathcal{L}_{i, i}=\left(\mathcal{L}_{V}\langle\rangle,\right)\left(e_{i}, e_{i}\right)$ and $\mathcal{L}_{i, N}=\left(\mathcal{L}_{V}\langle\rangle,\right)\left(e_{i}, N\right)$ for the Lie derivatives. First of all we notice that

$$
e_{i} e_{i}(g)=\left\langle D_{e_{i}} D_{e_{i}} N, V\right\rangle+2\left\langle D_{e_{i}} N, D_{e_{i}} V\right\rangle+\left\langle D_{e_{i}} D_{e_{i}} V, N\right\rangle \text {. }
$$

Because $-D_{e_{i}} N(p)=A\left(e_{i}(p)\right)=\lambda_{i} e_{i}(p)$, we have $\left\langle D_{e_{i}} N, D_{e_{i}} V\right\rangle(p)=-\frac{\lambda_{i}}{2} \mathcal{L}_{i, i}(p)$, which implies that

$$
e_{i} e_{i}(g)(p)=\left\langle D_{e_{i}} D_{e_{i}} N, V\right\rangle(p)+\left\langle D_{e_{i}} D_{e_{i}} V, N\right\rangle(p)-\lambda_{i} \mathcal{L}_{i, i}(p)
$$

We differentiate $\left\langle D_{e_{i}} V, N\right\rangle+\left\langle D_{N} V, e_{i}\right\rangle=\mathcal{L}_{i, N}$ in the direction $e_{i}$ to obtain the following identity:

$$
\left\langle D_{e_{i}} D_{e_{i}} V, N\right\rangle+\left\langle D_{e_{i}} V, D_{e_{i}} N\right\rangle+\left\langle D_{e_{i}} D_{N} V, e_{i}\right\rangle+\left\langle D_{N} V, D_{e_{i}} e_{i}\right\rangle=e_{i}\left(\mathcal{L}_{i, N}\right) .
$$

Using that $\left\{e_{1}, \ldots, e_{n}\right\}$ is a geodesic frame, one gets $\left(D_{e_{i}} e_{j}(p)\right)^{\top}=0$, from which we have $D_{e_{i}} e_{i}(p)=\lambda_{i} N(p)$. This yields

$$
\left\langle D_{N} V, D_{e_{i}} e_{i}\right\rangle(p)=\lambda_{i}\left\langle D_{N} V, N\right\rangle(p)=\frac{\lambda_{i}}{2} \mathcal{L}_{N, N}(p)
$$

Therefore, we have at $p$,

$$
\left\langle D_{e_{i}} D_{e_{i}} V, N\right\rangle=e_{i}\left(\mathcal{L}_{i, N}\right)+\frac{\lambda_{i}}{2} \mathcal{L}_{i, i}-\frac{\lambda_{i}}{2} \mathcal{L}_{N, N}-\left\langle D_{e_{i}} D_{N} V, e_{i}\right\rangle .
$$


On the other hand $\left[N, e_{i}\right](p)=D_{N} e_{i}(p)-D_{e_{i}} N(p)=-D_{e_{i}} N(p)=\lambda_{i} e_{i}(p)$, since $D_{N} e_{i}(p)=0$. Thus we infer that

$$
\left\langle D_{\left[N, e_{i}\right]} V, e_{i}\right\rangle(p)=\lambda_{i}\left\langle D_{e_{i}} V, e_{i}\right\rangle(p)=\frac{\lambda_{i}}{2} \mathcal{L}_{i, i}(p) .
$$

We now differentiate both sides of $\left\langle D_{e_{i}} V, e_{i}\right\rangle=\frac{1}{2} \mathcal{L}_{i, i}$ with respect to $N$ to obtain

$$
\left\langle D_{N} D_{e_{i}} V, e_{i}\right\rangle+\left\langle D_{e_{i}} V, D_{N} e_{i}\right\rangle=\frac{1}{2} N\left(\mathcal{L}_{i, i}\right) .
$$

Then $\left\langle D_{N} D_{e_{i}} V, e_{i}\right\rangle(p)=\frac{1}{2} N\left(\mathcal{L}_{i, i}\right)(p)$. But the definition of curvature yields

$$
\left\langle R\left(N, e_{i}\right) V, e_{i}\right\rangle(p)=\left\langle D_{e_{i}} D_{N} V, e_{i}\right\rangle(p)-\frac{1}{2} N\left(\mathcal{L}_{i, i}\right)(p)+\frac{\lambda_{i}}{2} \mathcal{L}_{i, i}(p) .
$$

We now substitute (3.3) in (3.2) and then substitute the result in (3.1) to obtain

$$
\begin{aligned}
e_{i} e_{i}(g)(p) & =-\left\langle R\left(N, e_{i}\right) V, e_{i}\right\rangle(p)+\left\langle D_{e_{i}} D_{e_{i}} N, V\right\rangle(p) \\
& +e_{i}\left(\mathcal{L}_{i, N}\right)(p)-\frac{1}{2} N\left(\mathcal{L}_{i, i}\right)(p)-\frac{\lambda_{i}}{2} \mathcal{L}_{N, N}(p) .
\end{aligned}
$$

Writing $V=\sum_{j=1}^{n} v_{j} e_{j}+g N$, where $v_{j}=\left\langle V, e_{j}\right\rangle$, we have

$$
\begin{aligned}
\left\langle D_{e_{i}} D_{e_{i}} N, V\right\rangle & =\left\langle D_{e_{i}} D_{e_{i}} N, \sum_{j=1}^{n} v_{j} e_{j}+g N\right\rangle \\
& =\sum_{j=1}^{n} v_{j}\left\langle D_{e_{i}} D_{e_{i}} N, e_{j}\right\rangle+g\left\langle D_{e_{i}} D_{e_{i}} N, N\right\rangle
\end{aligned}
$$

Taking the second covariant derivative of $\langle N, N\rangle$ in the direction $e_{i}$, we obtain $\left\langle D_{e_{i}} D_{e_{i}} N, N\right\rangle(p)=-\lambda_{i}^{2}$. We do the same for $\left\langle N, e_{j}\right\rangle$ to obtain

$$
\left\langle D_{e_{i}} D_{e_{i}} N, e_{j}\right\rangle+2\left\langle D_{e_{i}} N, D_{e_{i}} e_{j}\right\rangle+\left\langle N, D_{e_{i}} D_{e_{i}} e_{j}\right\rangle=0 .
$$

Therefore

$$
\left\langle D_{e_{i}} D_{e_{i}} N, e_{j}\right\rangle(p)=-\left\langle D_{e_{i}} D_{e_{i}} e_{j}, N\right\rangle(p) .
$$

Because $\left\langle D_{e_{i}} e_{j}, N\right\rangle=\left\langle D_{e_{j}} e_{i}, N\right\rangle$, we differentiate both sides in the direction $e_{i}$ to find

$$
\left\langle D_{e_{i}} D_{e_{i}} e_{j}, N\right\rangle+\left\langle D_{e_{i}} e_{j}, D_{e_{i}} N\right\rangle=\left\langle D_{e_{i}} D_{e_{j}} e_{i}, N\right\rangle+\left\langle D_{e_{j}} e_{i}, D_{e_{i}} N\right\rangle
$$

This equality, together with (3.4), allows us to write

$$
-\left\langle D_{e_{i}} D_{e_{i}} N, e_{j}\right\rangle(p)=\left\langle D_{e_{i}} D_{e_{i}} e_{j}, N\right\rangle(p)=\left\langle D_{e_{i}} D_{e_{j}} e_{i}, N\right\rangle(p) .
$$

Again using the curvature tensor as well as $\left[e_{i}, e_{j}\right](p)=0$ and to $e_{j}\left\langle D_{e_{i}} e_{i}, N\right\rangle(p)=$ $\left\langle D_{e_{j}} D_{e_{i}} e_{i}, N\right\rangle(p)+\left\langle D_{e_{i}} e_{i}, D_{e_{j}} N\right\rangle(p)=\left\langle D_{e_{j}} D_{e_{i}} e_{i}, N\right\rangle(p)$, we conclude that

$$
\begin{aligned}
\left\langle D_{e_{i}} D_{e_{i}} N, e_{j}\right\rangle(p) & =\left\langle R\left(e_{i}, e_{j}\right) e_{i}, e_{j}, N\right\rangle(p)-\left\langle D_{e_{j}} D_{e_{i}} e_{i}, N\right\rangle(p) \\
& =\left\langle R\left(e_{i}, e_{j}\right) e_{i}, N\right\rangle(p)-e_{j}\left\langle D_{e_{i}} e_{i}, N\right\rangle(p)
\end{aligned}
$$


From this we arrive at

$$
\begin{aligned}
\left\langle D_{e_{i}} D_{e_{i}} N, V\right\rangle(p) & =\sum_{j=1}^{n} v_{j}\left(\left\langle R\left(e_{i}, e_{j}\right) e_{i}, N\right\rangle(p)-e_{j}\left\langle D_{e_{i}} e_{i}, N\right\rangle(p)\right)-g(p) \lambda_{i}^{2} \\
& =\sum_{j=1}^{n} v_{j}\left\langle R\left(e_{i}, e_{j}\right) e_{i}, N\right\rangle(p)-\sum_{j=1}^{n} v_{j} e_{j}\left\langle D_{e_{i}} e_{i}, N\right\rangle(p)-g(p) \lambda_{i}^{2} \\
& =\left\langle R\left(e_{i}, \sum_{j=1}^{n} v_{j} e_{j}\right) e_{i}, N\right\rangle(p)-\sum_{j=1}^{n} v_{j} e_{j}\left\langle D_{e_{i}} e_{i}, N\right\rangle(p)-g(p) \lambda_{i}^{2} \\
& =\left\langle R\left(e_{i}, V-g N\right) e_{i}, N\right\rangle(p)-\sum_{j=1}^{n} v_{j} E_{j}\left\langle D_{e_{i}} e_{i}, N\right\rangle(p)-g(p) \lambda_{i}^{2} \\
& =\left\langle R\left(e_{i}, V\right) e_{i}, N\right\rangle(p)-g(p)\left\langle R\left(e_{i}, N\right) e_{i}, N\right\rangle(p)-g(p) \lambda_{i}^{2} \\
& -\sum_{j=1}^{n} v_{j} e_{j}\left\langle D_{e_{i}} e_{i}, N\right\rangle(p),
\end{aligned}
$$

which yields

$$
\begin{aligned}
\left\langle D_{e_{i}} D_{e_{i}} N, V\right\rangle(p)-\left\langle R\left(e_{i}, V\right) e_{i}, N\right\rangle(p) & =-g(p)\left\langle R\left(e_{i}, N\right) e_{i}, N\right\rangle(p)-g(p) \lambda_{i}^{2} \\
& -\sum_{j=1}^{n} v_{j} e_{j}\left\langle D_{e_{i}} e_{i}, N\right\rangle(p) .
\end{aligned}
$$

We now make use of the identity obtained for $e_{i} e_{i}(g)$ to conclude that

$$
\begin{aligned}
e_{i} e_{i}(g)(p) & =e_{i}\left(\mathcal{L}_{i, N}\right)(p)-\frac{1}{2} N\left(\mathcal{L}_{i, i}\right)(p)-\frac{\lambda_{i}}{2} \mathcal{L}_{N, N}(p)-g(p) \lambda_{i}^{2} \\
& -g(p)\left\langle R\left(e_{i}, N\right) e_{i}, N\right\rangle(p)-\sum_{l=1}^{n}\left\langle V, e_{l}\right\rangle\left(e_{l}\left\langle A\left(e_{i}\right), e_{i}\right\rangle\right)(p) .
\end{aligned}
$$

It is convenient to note that

$$
\begin{aligned}
L_{r}(g)(p) & =\operatorname{tr}\left(P_{r} \circ \text {Hess }_{g}\right)(p)=\sum_{i=1}^{n}\left\langle P_{r} \operatorname{Hess}_{g}\left(e_{i}\right), e_{i}\right\rangle(p) \\
& =\sum_{i=1}^{n}\left\langle P_{r}\left(D_{e_{i}} \nabla g\right), e_{i}\right\rangle(p)=\sum_{i=1}^{n} \frac{\partial S_{r+1}}{\partial \lambda_{i}}\left\langle D_{e_{i}} \nabla g, e_{i}\right\rangle(p) .
\end{aligned}
$$

Hence, writing $\nabla g=\sum_{i=1}^{n} g_{j} e_{j}$, we find

$$
D_{e_{i}} \nabla g(p)=\sum_{j=1}^{n}\left(g_{j i} e_{j}+g_{j} D_{e_{i}} e_{j}\right)(p)=\sum_{j=1}^{n} g_{j i} e_{j}(p),
$$

which gives us $L_{r}(g)(p)=\sum_{i=1}^{n} \frac{\partial S_{r+1}}{\partial \lambda_{i}} g_{i i}(p)$. 
Moreover, taking into account that the curvature of a space form $M_{c}^{n+1}$ is given by $R(X, Y)=c(X \wedge Y)$ and that $\left(\mathcal{L}_{V}\langle\rangle,\right)(X, Y)=2 \psi\langle X, Y\rangle$, we deduce that

$$
\begin{aligned}
L_{r}(g)(p) & =-\psi(p) \sum_{i=1}^{n} \lambda_{i} \frac{\partial S_{r+1}}{\partial \lambda_{i}}-N(\psi)(p) \sum_{i=1}^{n} \frac{\partial S_{r+1}}{\partial \lambda_{i}}-c g(p) \sum_{i=1}^{n} \frac{\partial S_{r+1}}{\partial \lambda_{i}} \\
& -g(p) \sum_{i=1}^{n} \lambda_{i}^{2} \frac{\partial S_{r+1}}{\partial \lambda_{i}}-\sum_{l=1}^{n}\left\langle V, e_{l}\right\rangle\left(e_{l}\left\langle A\left(e_{i}\right), e_{i}\right\rangle\right)(p) \\
& =-(r+1) S_{r+1} \psi(p)-(n-r) S_{r} N(\psi)(p)-c(n-r) S_{r} g(p) \\
& -\left(S_{1} S_{r+1}-(r+2) S_{r+2}\right) g(p)-I .
\end{aligned}
$$

We now notice that

$$
\begin{aligned}
\operatorname{tr}\left(P_{r} D_{X} A\right)(p) & =\sum_{i=1}^{n}\left\langle\left(P_{r} D_{X} A\right)\left(e_{i}\right), e_{i}\right\rangle(p) \\
& =\sum_{i=1}^{n}\left\langle P_{r}\left(\left(D_{X} A\right)\left(e_{i}\right)\right), e_{i}\right\rangle(p) \\
& =\sum_{i=1}^{n}\left\langle\left(D_{X} A\right)\left(e_{i}\right), P_{r}\left(e_{i}\right)\right\rangle(p) \\
& =\sum_{i=1}^{n} \frac{\partial S_{r+1}}{\partial \lambda_{i}}\left\langle\left(D_{X} A\right)\left(e_{i}\right)-A\left(D_{X} e_{i}\right), e_{i}\right\rangle(p) .
\end{aligned}
$$

On the other hand, letting $X=e_{l}$ in the above equality and using $D_{e_{l}} e_{i}(p)=0$, one gets

$$
\left\langle e_{l}, \nabla S_{r+1}\right\rangle(p)=\operatorname{tr}\left(P_{r} D_{e_{l}} A\right)(p)=\sum_{i=1}^{n} \frac{\partial S_{r+1}}{\partial \lambda_{i}}\left\langle D_{e_{l}} A\left(e_{i}\right), e_{i}\right\rangle(p) .
$$

Finally we compute

$$
e_{l}\left\langle A\left(e_{i}\right), e_{i}\right\rangle(p)=\left\langle D_{e_{l}} A\left(e_{i}\right), e_{i}\right\rangle(p)+\left\langle A\left(e_{i}\right), D_{e_{l}} e_{i}\right\rangle(p)=\left\langle D_{e_{l}} A\left(e_{i}\right), e_{i}\right\rangle(p)
$$

to deduce that

$$
I=\sum_{l=1}^{n}\left\langle V, e_{l}\right\rangle\left\langle e_{l}, \nabla S_{r+1}\right\rangle(p)=\left\langle V, \nabla S_{r+1}\right\rangle(p) .
$$

From the above one gets

$$
\begin{aligned}
L_{r}(g) & =-\left(S_{1} S_{r+1}-(r+2) S_{r+2}\right) g-c(n-r) S_{r} g \\
& -(n-r) S_{r} N(\psi)-(r+1) S_{r+1} \psi-\left\langle V, \nabla S_{r+1}\right\rangle,
\end{aligned}
$$

which concludes the proof of the theorem.

Now we combine Corollary 2.3 and formula (8.4) of Alias et al. 3] to derive the following lemma.

Lemma 3.2. Let $x: M^{n} \rightarrow M_{c}^{n+1}$ be an oriented isometric immersion with unit normal vector field $N$. If $V$ is a conformal vector field on $M_{c}^{n+1}$ with conformal factor $\psi$ and $g=\langle V, N\rangle$ represents the support function on $M^{n}$, then

$$
\operatorname{div}\left(P_{r}\left(V^{T}\right)\right)=(n-r) S_{r} \psi+(r+1) S_{r+1} g .
$$

Putting together Theorem 3.1 and Lemma 3.2 we derive the next corollary. 
Corollary 3.3. Let $x: M^{n} \rightarrow M_{c}^{n+1}$ be an oriented isometric immersion with $S_{r+1}$ constant. If $V=s(d) \nabla d, g=\langle V, N\rangle$ and $W=(n-r-1) P_{r} \nabla g+(r+1) P_{r+1}\left(V^{T}\right)$, then

$$
\operatorname{div}(W)=-n(r+2)\left(\begin{array}{c}
n \\
r+2
\end{array}\right)\left(H_{1} H_{r+1}-H_{r+2}\right) g
$$

where $N$ is a unit normal vector field to $M^{n}$ and $d$ is the distance on $M_{c}^{n+1}$ to a fixed point $p_{0}$.

Proof. First we notice that $V=s(d) \nabla d$ is a conformal vector field with factor $\psi=s^{\prime}(d)$. Moreover, $N(\psi)=-c g$. On the other hand, since $S_{r+1}$ is constant Theorem 3.1 yields

$(n-r-1) L_{r}(g)=-(n-r-1)(r+1) S_{r+1} s^{\prime}(d)-(n-r-1)\left(S_{1} S_{r+1}-(r+2) S_{r+2}\right) g$.

We now use Lemma 3.2 to find

$$
(r+1) \operatorname{div}\left(P_{r+1}\left(V^{T}\right)\right)=(n-r-1)(r+1) S_{r+1} s^{\prime}(d)+(r+1)(r+2) S_{r+2} g .
$$

Therefore, using $L_{r}(g)=\operatorname{div}\left(P_{r} \nabla g\right)$ and $W=(n-r-1) P_{r} \nabla g+(r+1) P_{r+1}\left(V^{T}\right)$, we infer that

$$
\operatorname{div}(W)=-(n-r-1) S_{1} S_{r+1} g+n(r+2) S_{r+2} g .
$$

In order to deduce the desired result it is enough to notice that $S_{r}=\left(\begin{array}{l}n \\ r\end{array}\right) H_{r}$ and $(n-r-1) n\left(\begin{array}{c}n \\ r+1\end{array}\right)=n(r+2)\left(\begin{array}{c}n \\ r+2\end{array}\right)$.

\section{COMPaCt RADial GRAPHS}

By a compact smooth radial graph $\Sigma^{n} \subset \mathbb{R}^{n+1}$ we mean a differentiable graph whose domain is a full Euclidean sphere $\mathbb{S}^{n}(r)$ of radius $r>0$. In order to construct such a graph we fix a point $p_{0} \in \mathbb{R}^{n+1}$ called the origin, which coincides with the center of the sphere, and for each direction $v \in T_{p_{0}} \mathbb{R}^{n+1} \cong \mathbb{R}^{n+1}$ we consider a point $p(v) \in \Sigma^{n}$ that corresponds to the end point of a nontrivial geodesic segment on $\mathbb{R}^{n+1}$ starting from $p_{0}$ in the direction of $v$. We also call this type of graph a smooth star-shaped hypersurface of the Euclidean space $\mathbb{R}^{n+1}$.

A similar construction can be done for a compact smooth radial graph $\Sigma^{n} \subset$ $\mathbb{S}^{n+1}$. In fact, we fix a point $p_{0} \in \mathbb{S}^{n+1}$ and for each direction $v \in T_{p_{0}} \mathbb{S}^{n+1}$ we consider a point $p(v) \in \Sigma^{n}$ that corresponds to the end point of a nontrivial geodesic segment on $\mathbb{S}^{n+1}$ starting from $p_{0}$ in the direction of $v$.

Now we consider a compact smooth radial graph $\Sigma^{n} \subset \mathbb{R}^{n+1}$ whose domain is a Euclidean sphere $\mathbb{S}^{n}(r)$ of radius $r>0$. Next, we introduce local coordinates $u=$ $\left(u_{1}, \ldots, u_{n}\right)$ and let $X(u)$ and $Y(u)$ be parametrizations of $\mathbb{S}^{n}(r)$ and $\Sigma^{n}$ respectively. If $\rho(u)=|Y(u)|>0$, then $Y=\rho X$.

Let $f: \Sigma^{n} \rightarrow \mathbb{R}$ be the function defined by $f(Y)=\left\langle Y, N_{Y}\right\rangle$, where $N_{Y}$ is a unit vector field normal to $\Sigma^{n}$. Letting $\frac{\partial h}{\partial u_{i}}=h_{i}$, we have $Y_{i}=\rho X_{i}+\rho_{i} X$, from which we obtain

$$
\left\langle\rho X, \frac{Y_{1} \wedge \ldots \wedge Y_{n}}{\left|Y_{1} \wedge \ldots \wedge Y_{n}\right|}\right\rangle=\left\langle\rho X, \frac{\left(\rho X_{1}\right) \wedge \ldots \wedge\left(\rho X_{n}\right)}{\left|Y_{1} \wedge \ldots \wedge Y_{n}\right|}\right\rangle .
$$


Hence we deduce that

$$
\begin{aligned}
f(Y) & =\left\langle\rho X, \frac{Y_{1} \wedge \ldots \wedge Y_{n}}{\left|Y_{1} \wedge \ldots \wedge Y_{n}\right|}\right\rangle=\rho^{n+1} \frac{\left|X_{1} \wedge \ldots \wedge X_{n}\right|}{\left|Y_{1} \wedge \ldots \wedge Y_{n}\right|}\left\langle X, N_{X}\right\rangle \\
& =-\frac{\rho^{n+1}}{r} \frac{\left|X_{1} \wedge \ldots \wedge X_{n}\right|}{\left|Y_{1} \wedge \ldots \wedge Y_{n}\right|}\langle X, X\rangle=-r \rho^{n+1} \frac{\left|X_{1} \wedge \ldots \wedge X_{n}\right|}{\left|Y_{1} \wedge \ldots \wedge Y_{n}\right|}<0 .
\end{aligned}
$$

Here we are considering $N_{X}=-\frac{1}{r} X$ as the unit normal vector field to $\mathbb{S}^{n}(r)$ in such a way that its mean curvature is positive.

Finally, given a compact smooth radial graph $\Sigma^{n} \subset \mathbb{S}^{n+1}$ as above, we consider the stereographic projection $\pi: \mathbb{S}^{n+1} \backslash\left\{p_{0}\right\} \rightarrow \mathbb{R}^{n+1}$ and let $V_{\mathbb{S}^{n+1}}$ be the position vector field with basis point $p_{0}$ in $\mathbb{S}^{n+1}$. Hence we have the next lemma.

Lemma 4.1. Under the above conditions the function $g=\left\langle V_{\mathbb{S}^{n+1}}, N_{Y}\right\rangle$ has a welldefined sign.

Proof. Let $X$ and $Y$ be parametrizations of $\mathbb{S}^{n}(r)$ and $\Sigma^{n}$ respectively. Then $\pi(X)$ is a parametrization of a sphere on $\mathbb{R}^{n+1}$ while $\pi(Y)$ is a parametrization of a smooth radial graph over $\pi(X)$ on $\mathbb{R}^{n+1}$.

Let $g_{1}, g_{2}: \pi(Y) \rightarrow \mathbb{R}$ be continuous functions such that either $g_{i}>0$ or $g_{i}<0$, $d \pi\left(V_{\mathbb{S}^{n+1}}\right)=g_{1} V_{\mathbb{R}^{n+1}}$ and $d \pi\left(N_{Y}\right)=g_{2} N_{\pi(Y)}$. If $e^{\phi}$ stands for the conformal factor of $\pi$, then we obtain

$$
e^{2 \phi}\left\langle V_{\mathbb{S}^{n+1}}, N_{Y}\right\rangle=\left\langle d \pi\left(V_{\mathbb{S}^{n+1}}\right), d \pi\left(N_{Y}\right)\right\rangle=\left\langle g_{1} V_{\mathbb{R}^{n+1}}, g_{2} N_{\pi(Y)}\right\rangle>0(\text { or }<0),
$$

from which we derive the desired result.

\section{Proof of Theorem 1.1}

Proof. Letting $r=1$ in Corollary 3.3 and integrating $\operatorname{div} W$ over $\Sigma^{n}$, we obtain

$$
\int_{\Sigma}\left(H_{1} H_{2}-H_{3}\right) g d \Sigma=0 .
$$

On the other hand, it is well known that for immersions $M^{n} \rightarrow M_{c}^{n+1}$,

$$
H_{r}^{2} \geq H_{r-1} H_{r+1}, \forall r \in\{1, \ldots, n-1\},
$$

with equality occurring if and only if $M$ is totally umbilical; see [6] or [1] for more details.

Taking into account that $S_{1}^{2}=|A|^{2}+2 S_{2}$, we see that $H_{1}^{2}>0$. Hence we may choose the orientation for $\Sigma^{n}$ in such a way that $H_{1}>0$. Since $H_{0}=1$ we obtain $H_{1}^{2} \geq H_{2}>0$. Now letting $r=2$ on (5.2) we have $-H_{1} H_{3} \geq-H_{2}^{2}$, from which we obtain

$$
H_{1}\left(H_{1} H_{2}-H_{3}\right) \geq H_{2}\left(H_{1}^{2}-H_{2}\right) \geq 0 .
$$

Since $g$ has a well-defined sign, we make use of equations (5.1) and (5.3) to arrive at $H_{1} H_{2}-H_{3}=0$. Thus taking this into account in inequality (5.3), we find that $H_{1}^{2}-H_{2}=0$. Hence we deduce that $x\left(M^{n}\right)$ is totally umbilical, which finishes the proof of the theorem.

For a compact smooth radial graph $\Sigma^{n} \subset \mathbb{S}^{n+1}$ of non-null constant mean curvature, we proved in 5 that $\Sigma^{n}$ is a round sphere. However, we avoided minimal graphs. In fact, this is easily extended to the minimal case according to the next theorem. 
Theorem 5.1. Let $\Sigma^{n} \subset \mathbb{S}^{n+1}$ be a compact smooth star-shaped minimal hypersurface. Then $\Sigma^{n}$ is totally geodesic.

Proof. Referring to Lemma 3.2, we have div $\left(P_{1}\left(V^{T}\right)\right)=2 S_{2} g$. This implies that

$$
\int_{\Sigma} S_{2} g d \Sigma=0
$$

Since $2 S_{2}=-|A|^{2} \leq 0$ and $g$ does not change sign, we get $S_{1}=S_{2}=0$. Therefore, we deduce that $|A|^{2}=0$, which yields that $\Sigma^{n}$ is totally geodesic.

\section{ACKNowledgement}

We would like to thank the referee for comments and valuable suggestions.

\section{REFERENCES}

1. Alias, L. and Colares, A.G., Uniqueness of spacelike hypersurfaces with constant higher order mean curvature in generalized Robertson-Walker spacetimes, Mathematical Proceedings of the Cambridge Philosophical Society, 143 (2007), 703-729. MR2373968 (2008m:53146)

2. Alias, L., Dajczer, M. and Ripoll, J., A Bernstein-type theorem for Riemannian manifolds with a Killing field, Ann. Global Anal. Geom., 31 (2007), 363-373. MR2325221|(2008i:53073)

3. Alias, L., de Lira, J. and Malacarne, J. M., Constant higher-order mean curvature hypersurfaces in Riemannian spaces, Jour. Inst. Math. Jussieu, 5 (2006), 527-562. MR2261223 (2007i:53062)

4. Barros, A., Brasil, A. and Caminha, A., Stability of spacelike hypersurfaces in foliated spacetimes, Diff. Geom. Appl., 26 (2008), 357-365. MR2423377

5. Barros, A. and Sousa, P., An extension of Jellett's theorem, Bull. Sci. Math., 133 (2009), 190-197.

6. Caminha, A., On spacelike hypersurfaces of constant sectional curvature Lorentz manifolds, Jour. Geom. Phys., 56 (2006), 1144-1174. MR2234043 (2007c:53092)

7. Fornari, S. and Ripoll, J., Killing fields, generalized Gauss map and constant mean curvature hypersurfaces. Illinois Jour. of Math., 48 (2004), 1385-1403. MR2114163 (2005i:53037)

8. Greene, R. and $\mathrm{Wu}, \mathrm{H}$., Function theory on manifolds which possess a pole, Lec. Notes in Math., 699, Springer, 1979. MR521983 (81a:53002)

9. Heintze, E., Extrinsic upper bounds for $\lambda_{1}$, Math. Ann., 280 (1988), 389-402. MR936318 (89f:53091)

10. Hsiung, C.C., Some integral formulas for closed hypersurfaces, Math. Scand., 2 (1954), 286294. MR0068236 (16:849j)

11. Jellett, J., La surface dont la courbure moyenne est constant, Jour. de Math. Pures et Appliquées, XVIII (1853), 163-167.

12. Montiel, S. and Ros, A., Compact hypersurfaces: The Alexandrov theorem for higher order mean curvatures, Differential geometry, A symposium in honour of Manfredo do Carmo, Pitman Monographs and Surveys in Pure and Applied Mathematics, 52, Longman, 1991, pp. 278-296. MR:1173047 (93h:53062)

13. Reilly, R., Variational properties of functions of the mean curvature for hypersurfaces in space form, J. Diff. Geom., 8 (1973), 465-477. MR0341351 (49:6102)

14. Ros, A., Compact hypersurfaces with constant scalar curvature and a congruence theorem, J. Diff. Geom., 27 (1988), 215-223. MR.925120 (89b:53096)

15. Ros, A., Compact hypersurfaces with constant higher order mean curvatures, Revista Matemática Iberoamericana, 3 (1987), 447-453. MR996826 (90c:53160)

16. Rosenberg, H., Hypersurfaces of constant curvature in space forms, Bull. Sci. Math., 117 (1993), 211-239. MR:1216008 (94b:53097) 
17. Sousa, P., O Laplaciano de uma função tipo suporte e aplicações, Master thesis, Univ. Federal do Ceará, 2003.

18. Suss, W., Uber Kennzeichnungen der Kugeln und Affinspharen durch Herrn K.-P. Grotemeyer, Arch. Math. (Basel), 3 (1952), 311-313. MR0053548 (14:789b)

Departamento de Matemática, Universidade Federal do Ceará, 60455-760 Fortaleza, BRAZIL

E-mail address: abbarros@mat.ufc.br

Departamento de Matemática, Universidade Federal do Piauí, 64049-550 Teresina, BRAZIL

E-mail address: pauloalexandre@ufpi.edu.br 